\title{
July 2014 Critical Care Case of the Month: There Is Still a Role for Physical Examination
}

\author{
Robert A. Raschke, MD
}

Banner Good Samaritan Medical Center

Phoenix, AZ

\section{History of Present IIIness}

A 90-year-old woman was the seatbelt-restrained driver in a low speed frontal motor vehicle collision with airbag deployment, after she accidentally hit the gas instead of the brake. In the emergency room, the patient's main complaint was right shoulder pain. On ER physical exam, she had sternal ecchymosis consistent with "seatbelt sign". Her right shoulder was said to be tender, but the mechanism of injury to the right shoulder was unclear since her drivers-side seatbelt would been in contact with her left rather than right shoulder. Her right upper extremity was said to be "weak secondary to pain". Further neurological examination was noted to be difficult due to "patient crying out in pain and anxiety", but it was noted that she could lift both legs off the bed. Her left knee was echymotic. Cardiac auscultation revealed irregularly irregular rhythm.

\section{PMH}

- Chronic atrial fibrillation

- Coronary artery disease

- Hypertension

\section{Medications}

- Warfarin

- Aspirin

- Clonidine

- Metoprolol

Labs performed in the emergency room showed an INR 1.9. Radiographs demonstrated a normal right shoulder and a left patellar fracture. CT scans of the cervical spine and chest showed no bony abnormalities. An incidental $4 \mathrm{~cm}$ thoracic aortic aneurysm was noted. CT of the brain showed periventricular white matter hyperlucencies consistent with small vessel disease. The patient became a bit drowsy after receiving narcotic analgesia in the emergency room and was transferred to the medical ICU for management of pain and delirium. 


\section{ICU Physical Examination}

In the medical ICU the patient was alert, and seemed much younger than 90 years of age, with a sharp wit. She complained of 10/10 shoulder pain at rest which occasionally made her wince, cry out in pain and move her shoulder however, she said there was no position in which her shoulder did not hurt. There were no ecchymosis of the shoulder, and it could be passively abducted and rotated without worsening the pain. The initial neurological examination was cursory and unrevealing because the patient was distracted by pain, and her left leg was immobilized. A short time later the nurse reported that she felt the patient's right leg was weak and the neurological exam was repeated. Strength in the patient's right leg was $1 / 5$, her left leg was immobilized, but ankle extension was $5 / 5$. She could not cooperate well with strength testing of her painful right arm, but her right grip was 2/5 with a normal strength in her left arm and hand. Toes were down-going and reflexes were generally hypoactive. She was not aphasic. Neurology was consulted.

Which of the following is true in regards to this patient's neurological findings?

1. A cervical spinal cord injury could explain these findings

2. A seat belt injury of the left carotid artery could have resulted in traumatic dissection and subsequent stroke

3. Right hemiparesis without aphasia could represent a lacunar stroke

4. They might represent a cardio-embolic stroke related to her history of atrial fibrillation

5. All of the above 


\section{Correct!}

\section{All the above.}

CT scans are poorly sensitive for cortical stroke in the first 24 hours. Lacunar strokes in the internal capsule typically cause hemiparesis but not aphasia (which is a cortical finding). Although we often consider spinal cord injuries as the cause of paraplegia or quadriplegia, a lateralized cord injury could cause a hemiparesis mimicking a stroke.

A CT angiogram of the neck was performed and demonstrated a focal $50 \%$ narrowing of the left common carotid artery (see below) compatible with, but not specific for an acute traumatic carotid dissection.

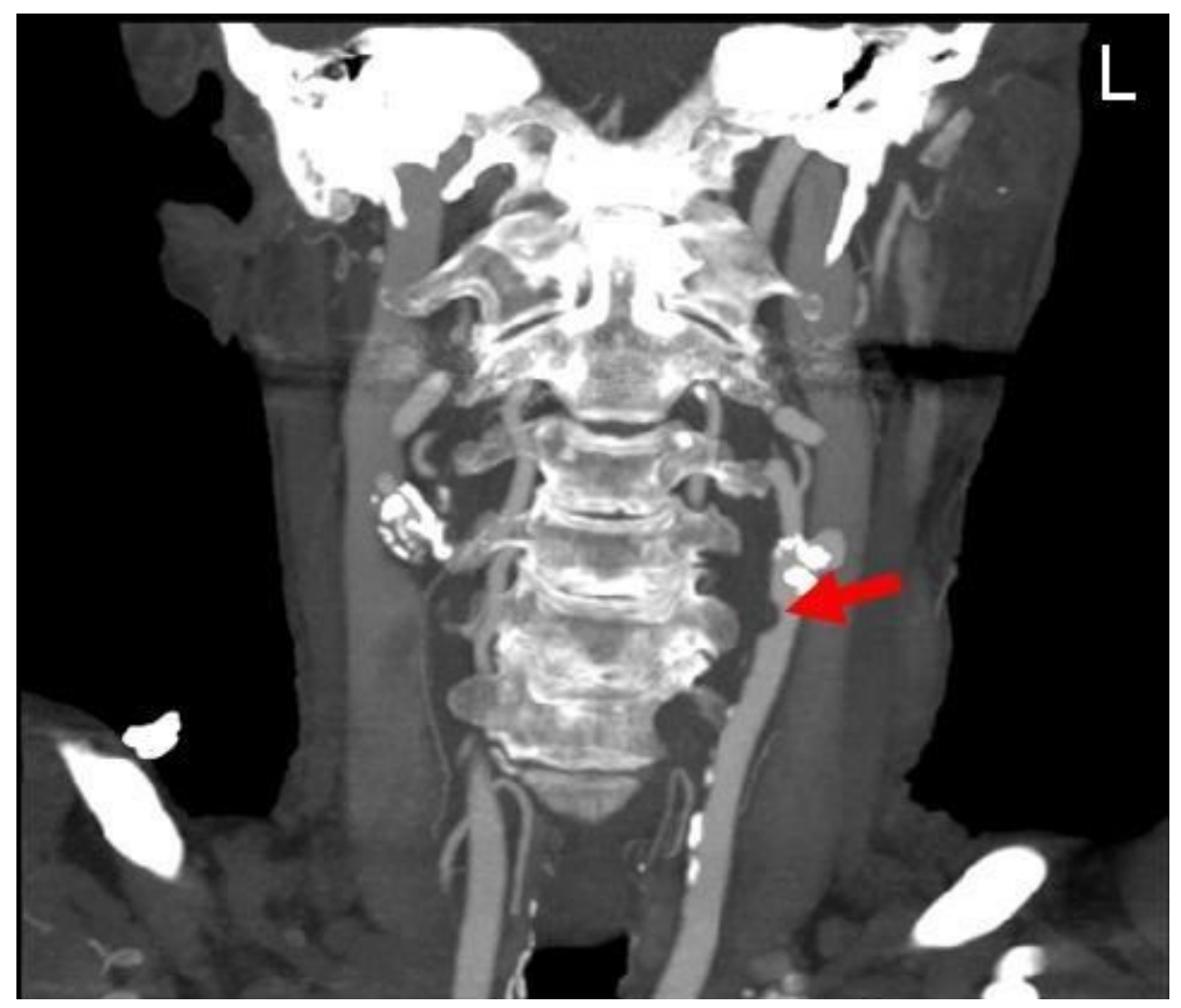

Figure 1. CT angiogram of the neck showing narrowing of the left common carotid artery (red arrow).

Carotid dissection is associated with trauma, often mild trauma, in about $40 \%$ of cases. It presents with headache or carotidynia in up to $90 \%$ of cases. A Horner's sign with ptosis and miosis may be seen on the affected side. Transient ischemic attacks and strokes are seen in about $25 \%$ and $50 \%$ of patients respectively. Thrombolytic therapy should not be withheld if indicated for patients who suffer stroke (and can be treated within the first 3 hours of symptoms). Anticoagulation or antiplatelet therapy is indicated in all patients. Endovascular procedures and vascular surgery are usually not needed. 
Aspirin was administered and vascular surgery consulted - although non-surgical management was planned. Shoulder pain persisted.

The next day, the patient complained of left upper extremity weakness. On examination 0/5 grip strength was noted bilaterally, with 4/5 shoulder abduction bilaterally.

What should be done next?

1. Repeat head CT

2. Begin heparin for an acute cerebral vascular accident (stroke)

3. Magnetic resonance imaging (MRI) of the neck

4. 1 and 3

5. All of the above 


\section{Correct!}

\section{1 and 3}

The neurological findings were interpreted as possibly consistent with an acute central cord syndrome due to cervical spondylotic myelopathy. Central cord syndrome (CCS) is an acute cervical spinal cord injury often associated with neck hyperextension in older persons with cervical spondylosis (1). It accounts for approximately $9 \%$ of traumatic spinal cord injuries. It classically presents with arm weakness worse than leg weakness, sensory loss and bowel and bladder dysfunction.

A MRI of the neck was performed (Figure 2).
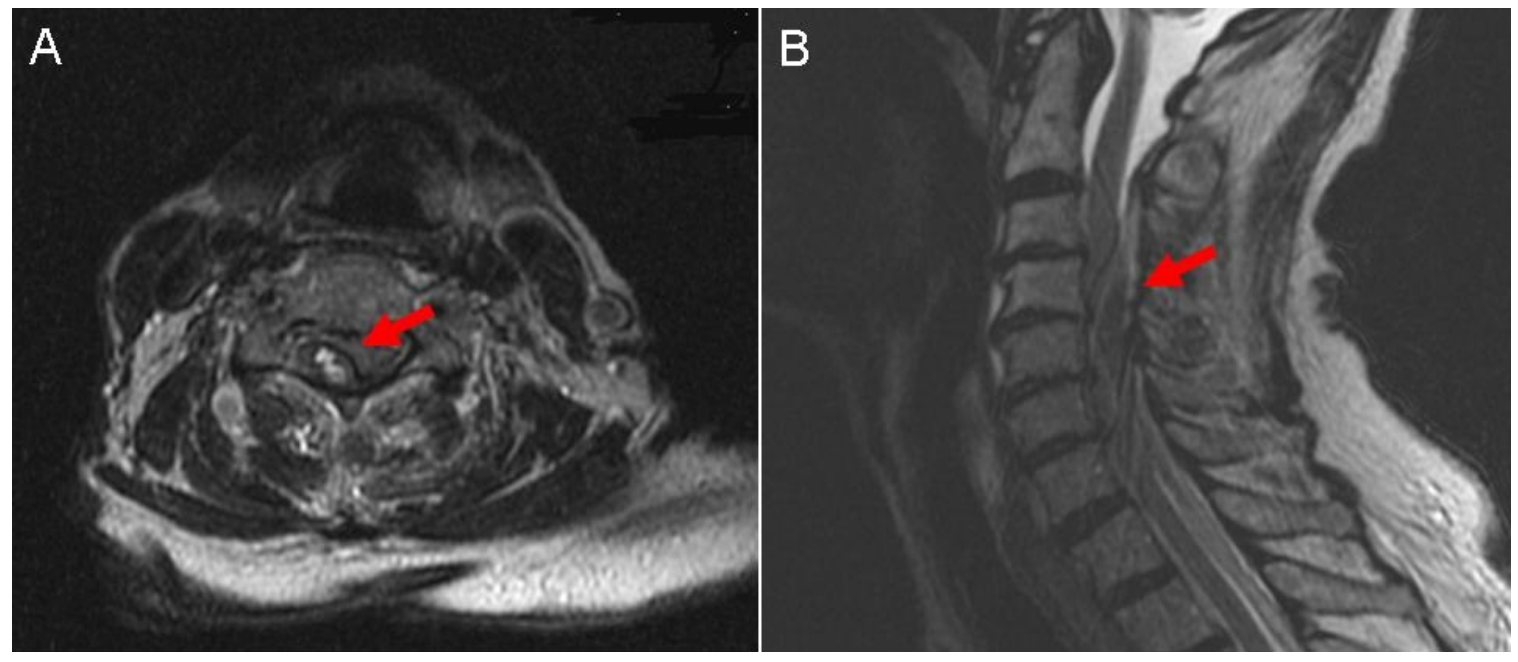

Figure 2. Panel A: axial view of the MRI of the spine. Panel B: saggital view. In both panels a right-sided epidural hematoma is demonstrated (red arrow) compressing the spinal column.

The scan did not show a central cord lesion with cervical spondylosis, but rather a right lateral epidural hematoma from C4-C6 with cord compression and cord edema.

Four units of fresh frozen plasma, 1500 units of prothrombin complex concentrate and $10 \mathrm{mg}$ vitamin $\mathrm{K}$ were administered intravenously, and the patient underwent emergent cervical decompressive laminectomy from C4-T1 with evacuation of the hematoma. The right shoulder pain immediately resolved and she has regained some grip strength post operatively, but is still early in her recovery. Vascular surgery opined that the CT angiogram findings described earlier likely were not due to carotid dissection.

Spinal epidural hematoma can be caused by trauma in a patient taking anticoagulation and antiplatelet drugs. It is usually caused by venous bleeding, and can therefore progress slowly. It presents with local and/or radicular pain, 
and loss of motor sensory and autonomic function below the level of the lesion. Lateral spinal epidural hematomas have been reported to cause Brown-Sequard syndrome (hemi-cord syndrome), and also to mimic stroke because they can cause hemiplegia, as in this case.

Which of the following is true?

1. Hyperreflexia classically associated with spinal cord lesions is typically a late finding.

2. The neurological examinations performed in this case were incomplete, yet serial examination was the major factor guiding care in the right direction.

3. The patient's unusual right shoulder pain was likely radicular, caused by the right-sided epidural hematoma at C4-C6.

4. When the patient only had right-sided weakness, sensory examination might have shown loss of pain on the left (classic finding of BrownSequard)

5. All of the above 


\section{Correct! \\ 5. All of the above}

In this case, many incorrect diagnoses were sequentially made:

1. Right shoulder musculoskeletal pain

2. Acute cardioembolic stroke

3. Left carotid dissection

4. Cervical spondylotic myelopathy with central cord syndrome

In each case, our physical examination was incomplete, but serial physical examination prompted rethinking of the differential diagnosis, eventually arriving at the correct and somewhat obscure explanation of the patient's symptoms and signs, leading to correct management.

Central cord syndrome is generally associated with a favorable prognosis for some degree of neurological and functional recovery. It can closely mimic stroke (2). The benefits of early surgical decompression in the setting of ATCCS remain controversial due to the lack of clinical randomized trials but recent studies suggest that early surgery (less than 72hours after trauma) appears to be safe and effective, especially for patients with evidence of focal anatomical cord compression (1).

The physical examination is difficult at times, and an ongoing job -it may not be complete after the initial history and physical examination. The patient's shoulder pain had some features to suggest that it was radicular rather than musculoskeletal. Anatomically a right C4-C6 epidural hematoma might be expected to cause right shoulder radicular pain. Perhaps more importantly, listen to the nurses. In this case, it was the nurses who noted the change in the neurological examination that led to the diagnosis.

\section{References}

1. Molliqaj G, Payer M, Schaller K, Tessitore E. Acute traumatic central cord syndrome: a comprehensive review. Neurochirurgie. 2014;60(1-2):5-11. [CrossRef] [PubMed]

2. Liou KC, Chen LA, Lin YJ. Cervical spinal epidural hematoma mimics acute ischemic stroke. Am J Emerg Med. 2012;30(7):1322.e1-3.[CrossRef] [PubMed] 